\title{
Title: Elucidation of Master Allostery Essential for Circadian Clock Oscillation in Cyanobacteria
}

\author{
Authors: Y. Furuike ${ }^{1,2, \dagger, *}$, A. Mukaiyama ${ }^{1,2, \dagger}$, D. Ouyang ${ }^{1}$, K. Ito-Miwa ${ }^{3}$, D. Simon ${ }^{1,2}$, E. \\ Yamashita $^{4}$, T. Kondo ${ }^{3}$, S. Akiyama ${ }^{1,2, *}$.
}

\begin{abstract}
Affiliations:
${ }^{1}$ Research Center of Integrative Molecular Systems (CIMoS), Institute for Molecular Science, National Institute of Natural Sciences, 38 Nishigo-Naka, Myodaiji, Okazaki 444-8585, Japan.

${ }^{2}$ Department of Functional Molecular Science, SOKENDAI (The Graduate University for Advanced Studies), 38 Nishigo-Naka, Myodaiji, Okazaki 444-8585, Japan.

${ }^{3}$ Division of Biological Science, Graduate School of Science and Institute for Advanced Studies, Nagoya University, 464-8602 Nagoya, Japan.

${ }^{4}$ Institute for Protein Research, Osaka University, 3-2 Yamada-oka, Suita 565-0871, Japan.
\end{abstract}

*Correspondence to: Y. Furuike (furuike@ims.ac.jp); S. Akiyama (akiyamas@ims.ac.jp)

$15 \quad †$ These authors contributed equally to this work.

Abstract: Spatio-temporal allostery is the source of complex but ordered biological phenomena. To identify the structural basis for allostery that drives the cyanobacterial circadian clock, we crystallized the clock protein KaiC in four distinct states, which cover a whole cycle of phosphortransfer events at Ser431 and Thr432. The minimal set of allosteric events required for oscillatory nature is a bidirectional coupling between the coil-to-helix transition of the Ser431-dependent phospho-switch in the C-terminal domain of KaiC and ADP release from its N-terminal domain during ATPase cycle. An engineered KaiC-protein oscillator consisting of a minimal set of the identified master allosteric events exhibited mono-phosphorylation cycle of Ser431 with a temperature-compensated circadian period, providing design principles for simple posttranslational biochemical circadian oscillators.

One Sentence Summary: Coupling between a phospho-switch and KaiC ATPase-dependent nucleotide exchange drives the cyanobacterial circadian clock.

Main Text: The cooperative nature of allosteric regulation is a source of nonlinearity that gives rise to oscillatory phenomena in diverse cellular functions $(1,2)$. Circadian clock systems are a typical example, wherein clock proteins are post-translationally phosphorylated at multiple sites in programmed (3-5) or pseudo-random manners $(6,7)$ as a means to allosterically regulate the stability of hetero-multimeric complexes of clock proteins $(8,9)$, delays for feedback loops $(6)$, and period length (10). Accordingly, a great deal of effort has been devoted to characterizing the phosphorylation-dependent allosteric structural changes in the clock proteins along the circadian reaction coordinate $(8,9,11-15)$. 
The cyanobacterium Synechococcus elongatus PCC7942 is one of the simplest prokaryotic model organisms (16). Only two adjacent amino acid residues, S431 and T432, in the core clock protein KaiC are phosphorylation targets $(17,18)$. In the presence of KaiA, KaiB, and ATP, KaiC exhibits a phosphorylation cycle in vitro (P-cycle): $\mathrm{ST} \rightarrow \mathrm{SpT} \rightarrow \mathrm{pSpT} \rightarrow \mathrm{pST} \rightarrow \mathrm{ST}$, where $\mathrm{S}$, $\mathrm{T}, \mathrm{pS}$, and pT indicate S431, T432, phosphorylated S431 (pS431), and phosphorylated T432 (pT432), respectively (17-19). Whereas the KaiC P-cycle has been investigated in vivo (20), in vitro $(19,21)$, and in silico $(18,22-24)$, little is known about the allosteric regulation of the KaiC P-cycle because all KaiC structures reported to date share nearly identical conformations at the phosphorylation sites, irrespective of the presence or absence of phosphoryl modifications $(8,9$, $14,15)$.

To visualize the structural basis for allosteric oscillatory regulation, we crystallized the KaiC hexamer in eight distinct states and sorted them from the fully phosphorylated KaiC-pSpT to the fully dephosphorylated KaiC-ST so that fractional changes in pS431 and pT432 per hexamer in the crystalline phase (Fig. 1A) reproduce those observed during the circadian cycle in solution (Fig. 1B). Each subunit of the KaiC hexamer consists of an N-terminal ATPase (C1) domain (11, 25) and a C-terminal auto-kinase/phosphatase (C2) domain $(14,21)$ (left panel of Fig. 1C). Dephosphorylation of pT432 in the fully KaiC-pSpT hexamer occurred in a stepwise manner, but no specific order was observed in regard to which subunit was dephosphorylated at each step (fig. $\mathrm{S} 1$ ). However, systematic ADP accumulation was observed for the C1 domain (C1-ADP) of KaiCpST (Fig. 1, A and C, and fig. S1). The accumulated C1-ADP molecules were replaced with C1ATP during the transition from KaiC-pST to KaiC-ST (Fig. 1C). These results indicate that the auto-dephosphorylation events in the $\mathrm{C} 2$ domain are linked to C1-ATP hydrolysis (ADP production) and $\mathrm{C} 1$-ADP exchange.

Our crystal-structure library indicates that key allosteric communication occurs between the $\mathrm{C} 1$ and $\mathrm{C} 2$ domains during the transition from KaiC-pST to KaiC-ST. We observed that the region upstream of S431 (T416-H429), which adopts a coiled structure in KaiC-pST, folds into a novel helical structure in KaiC-ST (left panels of Fig. 2). A helix-to-coil reversal was observed in the transition from KaiC-SpT to KaiC-pSpT (right panels of Fig. 2). These results enable us to assign the upstream region of S431 as a local phospho-switch (PSw). As shown in the left panels of Fig. 2, removal of the phosphoryl group from pS431 disrupted hydrogen bond interactions between pS431 and T426 in KaiC-pST, which provided the space necessary for the PSw to fold into the compact helical structure in KaiC-ST.

The PSw coil-to-helix transition in the $\mathrm{C} 2$ domain was coupled with the ADP release from the $\mathrm{C} 1$ domain. Indicated by arrows in Fig. 3A (gradient from orange to green), the ADP-to-ATP exchange at the $\mathrm{C} 1-\mathrm{C} 1$ interface resulted in a slight but systematic positional shift of the neighboring $\mathrm{C} 1$ domain. This rearrangement drove repositioning of the side chain of the basic residue R217 away from the neutral residue Q394 located in the C1-C2 interface (box in Fig. 3A), eventually disrupting the hydrogen bonding interaction between them that was observed in KaiCpST. Instead, the acidic residue E214 captured the released Q394 through a new hydrogen bond, thereby causing the $\mathrm{C} 2$ domain to move closer to the $\mathrm{C} 1$ domain in KaiC-ST. R393 and H429 are particular examples of this $\mathrm{C} 1$-directed movement by up to $\sim 3 \AA$, assisting the coil-to-helix switching of the PSw through multiple hydrogen-bond formation of S431 with I425 and S428 (upper left panel of Fig. 2).

The neutral character of residue 394 was critical to this allosteric communication because a Q394E mutation (KaiC ${ }^{\text {Q394E }}$ ) stabilized its electrostatic interaction with R217 but destabilized 
with E214, which resulted in constitutive phosphorylation, even with KaiC alone (Fig. 3B), and arrhythmic accumulation of its phosphorylated forms even in the presence of KaiA and KaiB (Fig. 3C). Consistent with this, KaiC ${ }^{\mathrm{Q} 34 \mathrm{~K}}$ exhibited very slow phosphorylation rates in the presence of KaiA (Fig. 3B) and remained dephosphorylated even in the presence of KaiA and KaiB (Fig. 3C). Hence, most of the large-scale bidirectional C1-C2 communication during the P-cycle takes place through an E214-R217-Q394 (ERQ) triad in the transition from KaiC-pST to KaiC-ST.

Our biochemical analysis demonstrated that the observed $\mathrm{C} 1-\mathrm{C} 2$ communication is necessary and sufficient to allosterically drive the P-cycle. We designed a KaiC T432V mutant (KaiC-SV) to investigate the mono-phosphorylation/dephosphorylation of S431 with minimal structural perturbation around residue 432. Valine is closest to threonine in terms of topology and volume (fig. S2, supplementary text), as evidenced by conservation of the helical PSw in KaiCSV (upper left panel of Fig. 2). Astonishingly, KaiC-SV exhibited an in vitro mono-P-cycle for S431 (Fig. 4A) (KaiC-SV $\leftrightarrow$ KaiC-pSV, red arrows in Fig. 2), an in vitro ATPase cycle (Fig. 4B), and an in vitro assembly and disassembly cycle (fig. S3, supplementary text) with a prolonged but temperature-compensated period $\left(51 \pm 2 \mathrm{~h}, Q_{10}=1.1\right)$ (Fig. 4C). By contrast, KaiC-CT, which was designed to allow for mono-phosphorylation/dephosphorylation of T432 with minimal structural perturbation around residue 431 , was arhythmic at $30^{\circ} \mathrm{C}$ (Fig. 4A). Furthermore, KaiC-SE, which was generated to allow for mono-phosphorylation/dephosphorylation of S431 without going through the fully-dephosphorylated KaiC-ST, was also arhythmic (Fig. 4A). The minimal set of allostery that ensures oscillatory nature is the coupling between the PSw transition associated with mono-phosphorylation/dephosphorylation of S431 and nucleotide exchange in the C1 domain (left panels of Fig. 2).

The prolonged mono-P-cycle observed for KaiC-SV implies that phosphorylated T432 (pT432) plays a role in accelerating the KaiC-ST P-cycle. In fact, the $\mathrm{O}_{\gamma}$ atom of S431 was positioned $1 \AA$ closer to C2-ATP in the KaiC-SpT-mimicking mutant KaiC-SE (upper right panel of Fig. 2A) relative to the position in KaiC-ST, which likely facilitates the subsequent formation of KaiC-pSpT. To investigate whether other mutations can allosterically suppress the periodprolonging T432V substitution, we introduced various ATPase-activating mutations into KaiCSV. A S157P substitution (KaiC ${ }^{\text {S157P }-S V), ~ w h i c h ~ i s ~ a ~ k n o w n ~ p e r i o d-s h o r t e n i n g ~ a n d ~ A T P a s e-~}$ activating mutation $(11,25,26)$, resulted in a less prolonged period $(40 \pm 2 \mathrm{~h})$ at $30^{\circ} \mathrm{C}$ (Fig. $\left.4 \mathrm{C}\right)$. We also introduced the ATPase-activating mutation D30A (27). The resultant $\mathrm{KaiC}^{\mathrm{S} 157 \mathrm{P}, \mathrm{D} 30 \mathrm{~A}-\mathrm{SV}}$ exhibited a near circadian mono-P-cycle $\left(29 \pm 2 \mathrm{~h}\right.$, Fig. 4D) at $35^{\circ} \mathrm{C}$ with a slight impairment in temperature compensation $\left(Q_{10}=1.4\right)$. However, it should be noted that the ATPase activities of

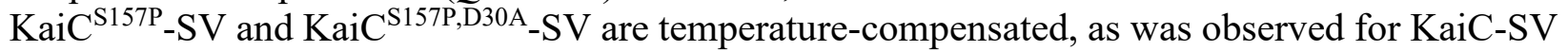
(Fig. 4C). Combined with KaiA and $\mathrm{KaiB}$, KaiC ${ }^{\mathrm{S} 157 \mathrm{P}, \mathrm{D} 30 \mathrm{~A}}-\mathrm{SV}$ constitutes a simple biochemical post-translational circadian oscillator with a sole-phosphorylation site.

The crucial role of the observed allostery is further supported by evolutional conservation and divergence of the dual phosphorylation sites. Multiple sequence alignment of KaiC and its homologues from other organisms revealed a near-perfect conservation of S431 (28). Only alanine (Fibrella aestuarina BUZ 2) and glycine (Allochromatium vinosum DSM 180) were observed exceptions, but the arrhythmicity of KaiC-AT and KaiC-GT in the presence of KaiA and KaiB supports our present interpretation (Fig. 4E). By contrast, amino acids other than threonine, such tyrosine (Pseudomonas oryzihabitans), histidine (Massilia sp. WG5), and proline (Pontibacter korlensis), are observed at position 432. We generated KaiC-SY, KaiC-SH, and KaiC-SP to investigate the effects of these substitutions and found that all of them exhibited arrhythmicity (Fig. 
4E). These results suggest that different evolutionary selection pressures acted on the two sites; position 431 was conserved to allow for acquisition of master $\mathrm{C} 1-\mathrm{C} 2$ allostery that gives rise to the temperature-compensated rhythmicity, and amino acid variances at position 432 fine-tune the period length into the circadian time scale by accelerating the C1-C2 allostery mediated by S431.

Mathematical modeling approaches have highlighted the importance of multisite phosphorylation in oscillatory phenomena. Simple enzymatic futile cycles in which a substrate shuttles between two states, e.g., mono-phosphorylated and dephosphorylated forms, via forward and backward reactions catalyzed by an opposing enzyme converge into an equilibrium (29). Unbiased systems targeting a substrate with two modification sites retain a limited $(\sim 0.1 \%)$ potential to exhibit self-sustained oscillation (30). This apparent inconsistency can be resolved by taking into consideration the fact that KaiC-SV can still be regarded as a two-site system because its single site for phosphorylation is influenced allosterically by another biochemically active site for ATP hydrolysis in the C1 domain (Fig. 3A).

The cyanobacterial circadian clock is the simplest circadian clock system known to date in terms of the number of components, but mechanistically it is a very complex system involving the ATPase cycle and a four-state P-cycle. The oscillators we designed using KaiC-SV and $\mathrm{KaiC}^{\mathrm{S} 157 \mathrm{P}, \mathrm{D} 30 \mathrm{~A}}-\mathrm{SV}$ reconstruct the complex system into a minimal unit by extracting the core allostery from the complex cycle. This will serve as a research tool for further elucidation of mechanisms, such as period determination, temperature compensability, and entrainment, and will provide design principles for the simplest post-translational biochemical oscillator that oscillate with a temperature-compensated circadian period. 


\section{References and Notes:}

1. A. Goldbeter, G. Dupont, Allosteric Regulation, Cooperativity, and Biochemical Oscillations. Biophys. Chem. 37, 341-353 (1990).

2. B. C. Goodwin, Oscillatory behavior in enzymatic control processes. Adv. Enzyme Regul. 3, 425-438 (1965).

3. R. Narasimamurthy et al., CK1 delta/epsilon protein kinase primes the PER2 circadian phosphoswitch. Proc. Natl. Acad. Sci. USA 115, 5986-5991 (2018).

4. R. Narasimamurthy, D. M. Virshup, The phosphorylation switch that regulates ticking of the circadian clock. Mol. Cell 81, 1133-1146 (2021).

5. C. L. Partch, Orchestration of Circadian Timing by Macromolecular Protein Assemblies. J. Mol. Biol. 432, 3426-3448 (2020).

6. A. Upadhyay, D. Marzoll, A. Diernfellner, M. Brunner, H. Herzel, Multiple random phosphorylations in clock proteins provide long delays and switches. Sci. Rep. 10, 22224 (2020).

7. A. C. R. Diernfellner, M. Brunner, Phosphorylation Timers in the Neurospora crassa Circadian Clock. J. Mol. Biol. 432, 3449-3465 (2020).

8. J. Snijder et al., Structures of the cyanobacterial circadian oscillator frozen in a fully assembled state. Science 355, 1181-1184 (2017).

9. R. Tseng et al., Structural basis of the day-night transition in a bacterial circadian clock. Science 355, 1174-1180 (2017).

10. H. Q. Yamaguchi, K. L. Ode, H. R. Ueda, A design principle for posttranslational chaotic oscillators. Iscience 24, 101946 (2021).

11. J. Abe et al., Atomic-scale origins of slowness in the cyanobacterial circadian clock. Science 349, 312-316 (2015).

12. R. P. Aryal et al., Macromolecular Assemblies of the Mammalian Circadian Clock. Mol. Cell 67, 770-782 (2017).

13. Y. Murayama et al., Tracking and visualizing the circadian ticking of the cyanobacterial clock protein KaiC in solution. EMBO J. 30, 68-78 (2011).

14. R. Pattanayek et al., Visualizing a circadian clock protein: Crystal structure of KaiC and functional insights. Mol. Cell 15, 841-841 (2004).

15. R. Pattanayek, Y. Xu, A. Lamichhane, C. H. Johnson, M. Egli, An arginine tetrad as mediator of input-dependent and input-independent ATPases in the clock protein KaiC. Acta Crystallog. D 70, 1375-1390 (2014).

16. M. Ishiura et al., Expression of a gene cluster kaiABC as a circadian feedback process in cyanobacteria. Science 281, 1519-1523 (1998).

17. T. Nishiwaki et al., A sequential program of dual phosphorylation of KaiC as a basis for circadian rhythm in cyanobacteria. EMBO J. 26, 4029-4037 (2007).

18. M. J. Rust, J. S. Markson, W. S. Lane, D. S. Fisher, E. K. O'Shea, Ordered phosphorylation governs oscillation of a three-protein circadian clock. Science 318, 809-812 (2007).

19. M. Nakajima et al., Reconstitution of circadian oscillation of cyanobacterial KaiC phosphorylation in vitro. Science 308, 414-415 (2005).

20. J. Tomita, M. Nakajima, T. Kondo, H. Iwasaki, No transcription-translation feedback in circadian rhythm of KaiC phosphorylation. Science 307, 251-254 (2005).

21. T. Nishiwaki et al., Role of KaiC phosphorylation in the circadian clock system of Synechococcus elongatus PCC 7942. Proc. Natl. Acad. Sci. USA 101, 13927-13932 (2004). 
22. T. S. Hatakeyama, K. Kaneko, Generic temperature compensation of biological clocks by autonomous regulation of catalyst concentration. Proc. Natl. Acad. Sci. USA 109, 81098114 (2012).

23. M. Sasai, Mechanism of autonomous synchronization of the circadian KaiABC rhythm. Sci. Rep. 11, 4713 (2021).

24. J. S. van Zon, D. K. Lubensky, P. R. H. Altena, P. R. ten Wolde, An allosteric model of circadian KaiC phosphorylation. Proc. Natl. Acad. Sci. USA 104, 7420-7425 (2007).

25. K. Terauchi et al., ATPase activity of KaiC determines the basic timing for circadian clock of cyanobacteria. Proc. Natl. Acad. Sci. USA 104, 16377-16381 (2007).

26. K. Ito-Miwa, Y. Furuike, S. Akiyama, T. Kondo, Tuning the circadian period of cyanobacteria up to 6.6 days by the single amino acid substitutions in KaiC. Proc. Natl. Acad. Sci. USA 117, 20926-20931 (2020).

27. D. Y. Ouyang et al., Development and Optimization of Expression, Purification, and ATPase Assay of KaiC for Medium-Throughput Screening of Circadian Clock Mutants in Cyanobacteria. Int. J. Mol. Sci. 20, 2789 (2019).

28. N. M. Schmelling et al., Minimal tool set for a prokaryotic circadian clock. BMC Evol. Biol. 17, 169 (2017).

29. D. Angeli, E. D. Sontag, Translation-invariant monotone systems, and a global convergence result for enzymatic futile cycles. Nonlinear Anal. Real World Appl. 9, 128140 (2008).

30. C. C. Jolley, K. L. Ode, H. R. Ueda, A Design Principle for a Posttranslational Biochemical Oscillator. Cell Rep. 2, 938-950 (2012). 
Acknowledgments: Diffraction data were collected at BL44XU at the SPring-8 facility under the proposals 2017A6700, 2017B6700, 2018A6700, 2018B6700, 2019A6700, 2019B6700, 2020A6700，2020A6500，2017A6702，2017B6702，2018A6802，2018B6802，2019A6902, 2019B6902, and 2020A6502. This research was partly supported by the Platform Project for Supporting Drug Discovery and Life Science Research (BINDS) from AMED under Grant Number JP20am0101072. Funding: This study was supported by Grants-in-Aid for Scientific Research (17H06165 to S.A.; 19K16061 to Y.F.; and 18K06171 to A.M.); Author contributions: Y.F., A.M., and S.A. designed the experiments. Y.F. collected the diffraction data and analyzed it with input from E.Y. Y.F., A.M., D.O., and D.S. performed the biochemical experiments. K.I.-M. and Y.F. performed in vivo experiments with input from T.K. S.A. and Y.F. drafted the manuscript with input from all authors. Competing interests: The authors declare no competing interests. Data and materials availability: The atomic coordinates and structure factors are deposited in the Protein Data Bank with accession codes 7DYK (KaiC-SV), 7DYJ (KaiC-ST), 7DY2 (KaiCpST), 7DYI (KaiC-SE), 7DXQ (KaiC-pSpT), and 7V3X (KaiC-pSpT \& pST). Requests for data or materials should be addressed to akiyamas@ims.ac.jp.

\section{Supplementary Materials:}

Materials and Methods

Figures S1-S3

Tables S1

References (31-40) 
A
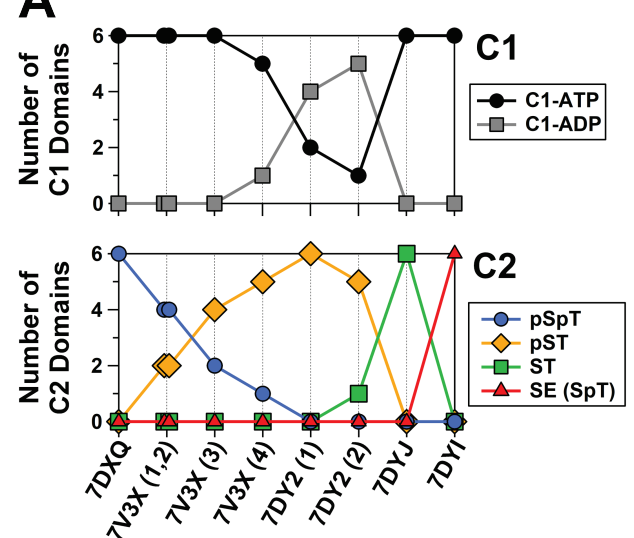

KaiC Hexamers in Crystal

B (PDB Accession Code)

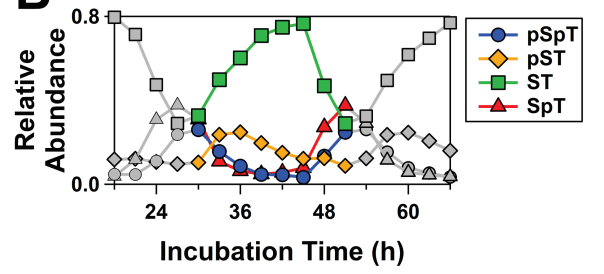

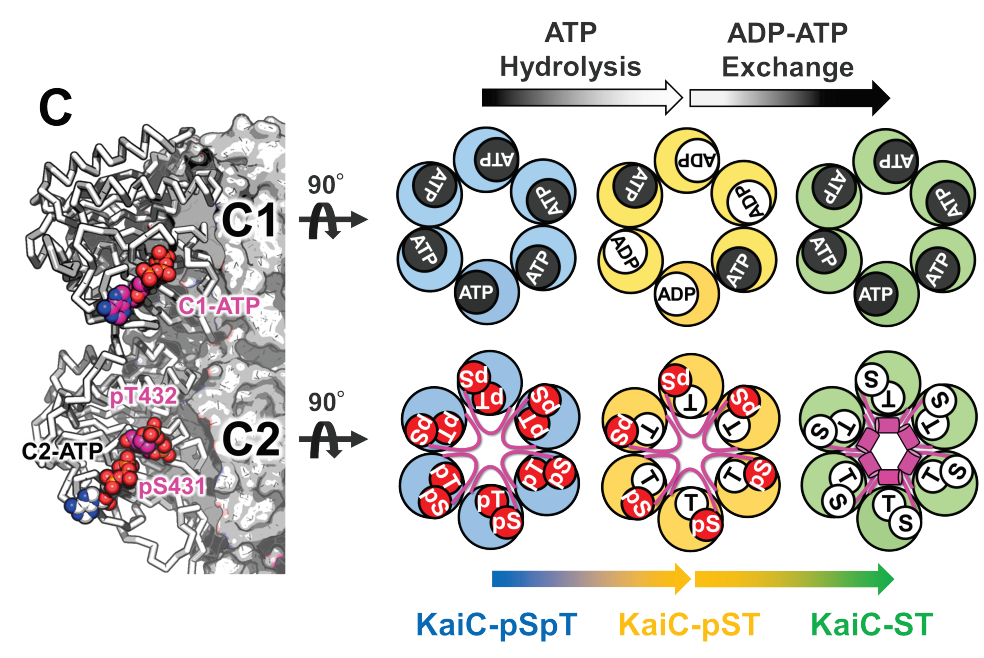

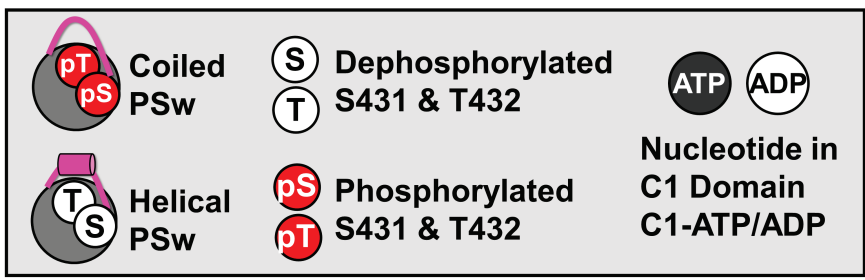

Fig. 1. KaiC crystal structures sorted by phosphorylation, ATP hydrolysis, and nucleotideexchange events. (A) Fractional changes for ATP/ADP bound in the C1 domain (upper panel) and phosphorylation status of S431 and T432 in the C2 domain (lower panel) in the crystalline phase. Horizontal axis depicts the PDB accession codes for nine KaiC hexamers crystallized in eight distinct states. For 7V3X and 7DY2, four and two hexamers present in each asymmetric unit are distinguished by the number in parentheses. Vertical axes represent the number of $\mathrm{C} 1$ and $\mathrm{C} 2$ domains that are in the ATP/ADP-bound and phosphorylated/dephosphorylated states, respectively, in the hexamer of interest. (B) Phosphorylation cycle for KaiC in solution. (C) Schematic drawings for three representative hexamers for KaiC-pSpT (7DXQ), KaiC-pST (7DY2), and KaiC-ST (7DYJ). Arrows above and below the hexameric rings represent biochemical reactions and state transitions, respectively, that are suggested by the sorted structures. The nomenclatures used in the drawings are defined in the bottom panel. 


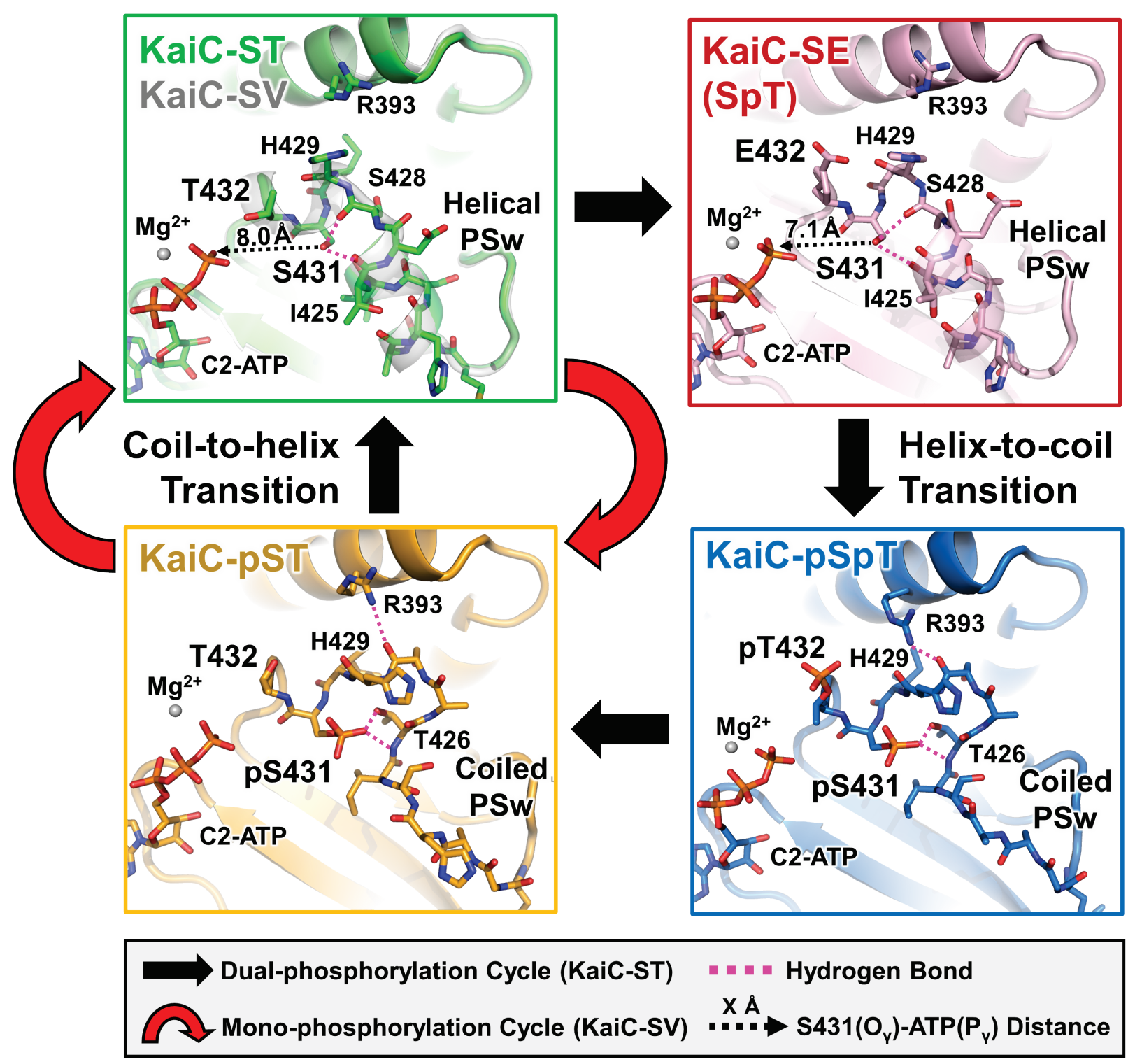

Fig. 2. Cyclic structural changes in the phosphor-switch (PSw) located upstream of S431 in KaiC-ST (green), KaiC-SV (white), KaiC-SE (pink), KaiC-pSpT (blue), and KaiC-pST (orange). Black and red arrows represent the dual- and mono-phosphorylation cycles in KaiC-ST and KaiC-SV, respectively. Dashed magenta lines correspond to hydrogen bonds around $\mathrm{S} 431 / \mathrm{pS} 431$. Dashed black arrows indicate the distances between the $\mathrm{S} 431 \mathrm{O}_{\gamma}$ atom and C2-ATP $\mathrm{P}_{\gamma}$ atom. 



Fig. 3. Structural basis for master allostery between C1-ATPase and C2-PSw in KaiC. (A) Tertiary and quaternary structural changes in the transition from KaiC-pST (orange) to KaiC-ST (green). The ADP-ATP exchange occurring at the $\mathrm{C} 1-\mathrm{C} 1$ interfaces and the coil-to-helix transition for PSw are allosterically coupled via rearrangements of E214, R217, Q394, R393, and H429. The inserted box indicates the zoomed-in-view of the ERQ triad mediating communication between the $\mathrm{C} 1$ and C2 domains. (B) Effects of Q394E and Q394K mutations on the switching ability of $\mathrm{C} 1-\mathrm{C} 2$ allostery. Proportion of phosphorylated KaiC (P-KaiC) was measured for $24 \mathrm{~h}$ at $30^{\circ} \mathrm{C}$ and after the KaiA addition at $24 \mathrm{~h}$. (C) Effects of Q394E and Q394K mutations on the P-cycle. 

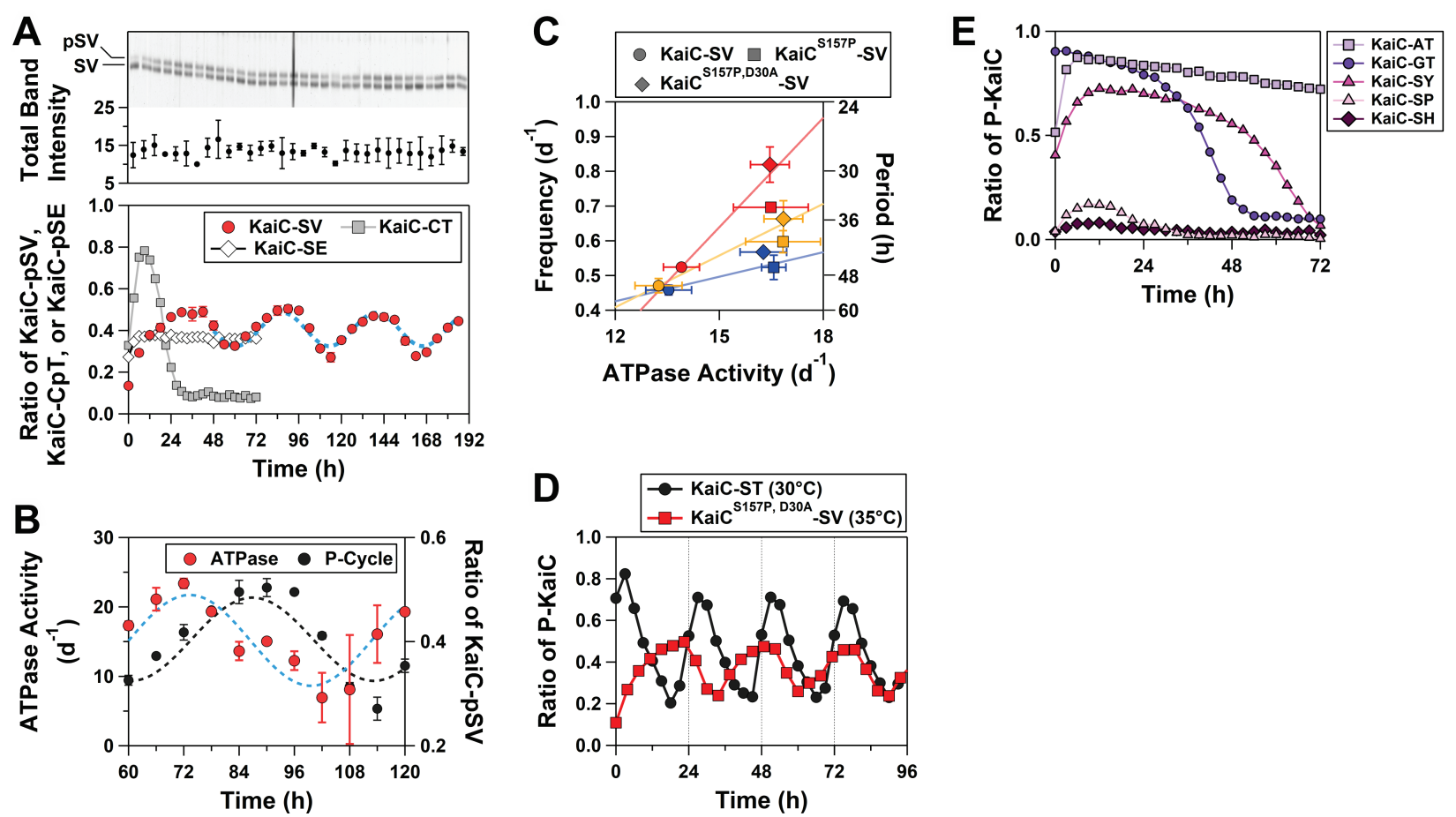

Fig. 4. Mono-phosphorylation rhythms for KaiC-SV. (A) In vitro P-cycle at $30^{\circ} \mathrm{C}$. Dotted line represents fitting of a cosine function to the data. Upper panel represents a magnified view of the upper phosphorylated (pSV) and lower dephosphorylated (SV) bands in SDS-PAGE gels. (B) In vitro ATPase rhythm at $30^{\circ} \mathrm{C}$. (C) Correlation plot between ATPase activity for KaiC alone and the frequency (24/period) of the in vitro P-cycle in the presence of KaiA and KaiB. Blue, orange, and red markers correspond to data analyzed at 25,30 , and $35^{\circ} \mathrm{C}$, respectively. (D) Near circadian mono-P-cycle $(29 \pm 2 \mathrm{~h})$ for $\mathrm{KaiC}^{\mathrm{S} 157 \mathrm{P}, \mathrm{D} 30 \mathrm{~A}}-\mathrm{SV}$ at $35^{\circ} \mathrm{C}$. (E) Arrhythmicity of KaiC-AT, KaiCGT, KaiC-SY, KaiC-SP, and $\mathrm{KaiC}-\mathrm{SH}$ at $30^{\circ} \mathrm{C}$. 